\title{
Homological algebra modulo exact zero-divisors
}

\author{
Petter Andreas Bergh, Olgur Celikbas, and David A. Jorgensen
}

\begin{abstract}
We study the homological behavior of modules over local rings modulo exact zero-divisors. We obtain new results which are in some sense "opposite" to those known for modules over local rings modulo regular elements.
\end{abstract}

\section{Introduction}

Given a local (meaning also commutative and Noetherian) ring $S$ and an ideal $I$, one may ask whether the homological behavior of modules over $S / I$ is related to that over $S$. In general this is hopeless; one needs to restrict the ideal $I$. When $I$ is generated by a regular sequence, there is a well-developed and powerful theory relating the homological properties of modules over these two rings (see for instance [Av2], [AvBu], [Be], [Dao1], [Eis], [Gu1], [Jo1], and [Jo2]). For example, suppose that the ideal $I$ is generated by a single regular element $x \in S$, denote the factor $\operatorname{ring} S /(x)$ by $R$, and let $M$ and $N$ be $R$-modules. Then a primary result is that

$$
\operatorname{Tor}_{1}^{R}(M, N)=\operatorname{Tor}_{2}^{R}(M, N)=\cdots=\operatorname{Tor}_{n}^{R}(M, N)=0,
$$

for some $n \geq 2$, implies that

$$
\operatorname{Tor}_{2}^{S}(M, N)=\cdots=\operatorname{Tor}_{n}^{S}(M, N)=0 .
$$

In other words, the vanishing of homology over $R$ implies the vanishing of homology over $S$. An analogous statement for cohomology also holds.

Another primary result compares the complexity of a finitely generated $R$ module with its complexity as an $S$-module (see Section 3 for the definition of complexity). Namely, there are inequalities (see [Av2, Remark 3.2(3)])

$$
\operatorname{cx}_{S}(M) \leq \operatorname{cx}_{R}(M) \leq \operatorname{cx}_{S}(M)+1 .
$$

In this paper we study the case where the element $x \in S$ is in some sense the "next best thing" to being a regular element. More precisely, we consider the case where the annihilator of $x$ is a nonzero principal ideal whose annihilator is also principal (and therefore is the ideal $(x)$ ). In accord with [HeS]], the element $x$ is said to be an exact zero-divisor if it is nonzero, it belongs to the maximal

Kyoto Journal of Mathematics, Vol. 54, No. 4 (2014), 879-895

DOI $10.1215 / 21562261-2801867$, (C) 2014 by Kyoto University

Received April 15, 2013. Revised November 12, 2013. Accepted November 28, 2013.

2010 Mathematics Subject Classification: Primary 13D03. 
ideal of $S$, and there exists another element $y \in S \operatorname{such}$ that $\operatorname{ann}_{S}(x)=(y)$ and $\operatorname{ann}_{S}(y)=(x)$. In this case we say that $(x, y)$ is a pair of exact zero-divisors of $S$. The ideal $(x)$ is then an example of a quasicomplete intersection ideal, a notion introduced in [AHŞ]. In that same paper, results relating certain invariants of modules over $S$ with those over $S /(x)$ are proved. We continue along similar, but more homological lines, and show that even if the element $x$ is the next best thing to being regular, namely, an exact zero-divisor, then the homological relationships between $S /(x)$-modules over $S /(x)$ and over $S$ change dramatically compared to the case where $x$ is regular. Two of our main results in Section 2 concern the vanishing of (co)homology. In particular, the result for homology takes the following form.

\section{THEOREM}

Let $R=S /(x)$ where $S$ is a local ring and $(x, y)$ is a pair of exact zero-divisors in $S$. Furthermore, let $M$ and $N$ be $R$-modules such that $y N=0$. If there exists an integer $n \geq 2$ such that $\operatorname{Tor}_{i}^{R}(M, N)=0$ for $1 \leq i \leq n$, then $\operatorname{Tor}_{i}^{S}(M, N) \cong$ $M \otimes_{S} N$ for $1 \leq i \leq n-1$.

Compared with the vanishing result for the case where $x$ is regular, the conclusion of the previous theorem is opposite in the sense that it is a nonvanishing result: the vanishing of homology over $R$ implies the nonvanishing of homology over $S$ (when the modules involved are nonzero and finitely generated). For cohomology, we obtain the following analogue.

\section{THEOREM}

Let $R=S /(x)$ where $S$ is a local ring and $(x, y)$ is a pair of exact zero-divisors in $S$. Furthermore, let $M$ and $N$ be $R$-modules such that $y N=0$. If there exists an integer $n \geq 2$ such that $\operatorname{Ext}_{R}^{i}(M, N)=0$ for $1 \leq i \leq n$, then $\operatorname{Ext}_{S}^{i}(M, N) \cong$ $\operatorname{Hom}_{S}(M, N)$ for $1 \leq i \leq n-1$.

We also compare the complexities of finitely generated modules over $R$ and over $S$. Similar to our previous results, we show that such a comparison is quite different from the case where $x$ is regular. The following theorem is the main result of Section 3.

\section{THEOREM}

Let $R=S /(x)$ where $S$ is a local ring and $x$ is an exact zero-divisor in $S$. If $M$ is a finitely generated $R$-module, then for any $n$ there are inequalities

$$
\beta_{n}^{R}(M)-\sum_{i=0}^{n-2} \beta_{i}^{R}(M) \leq \beta_{n}^{S}(M) \leq \sum_{i=0}^{n} \beta_{i}^{R}(M)
$$

of Betti numbers. In particular, the inequality $\mathrm{cx}_{S}(M) \leq \mathrm{cx}_{R}(M)+1$ holds. 
In the final section, Section 4, we discuss canonical endomorphisms of complexes of finitely generated free $R$-modules, and canonical elements of $\operatorname{Ext}_{R}^{2}(M, M)$, for finitely generated $R$-modules $M$, in the case where $R=S /(x)$ and $(x, x)$ is a pair of exact zero-divisors of $S$. The main result, Theorem 4.2, equates the ability to lift a finitely generated $R$-module $M$ from $R$ to $S$ to the triviality of the canonical element in $\operatorname{Ext}_{R}^{2}(M, M)$. This generalizes classical results (see, e.g., [ADS]) on lifting modules from $T /(x)$ to $T /\left(x^{2}\right)$ in the case where $x$ is a nonzero-divisor of the local ring $T$ (cf. Example 4.3 below).

\section{Vanishing results}

In this section we prove our vanishing results, starting with the homology version. We fix a local ring $S$ and a pair of exact zero-divisors $(x, y)$, and denote the local ring $S /(x)$ by $R$. It should be mentioned that the modules we consider in this section are not necessarily assumed to be finitely generated.

Since a (deleted) free resolution of $R$ over $S$ has the form

$$
\cdots \rightarrow S \stackrel{y}{\rightarrow} S \stackrel{x}{\rightarrow} S \stackrel{y}{\rightarrow} S \stackrel{x}{\rightarrow} S \rightarrow 0
$$

one has for any $R$-module $N$ the following:

$$
\operatorname{Tor}_{q}^{S}(R, N) \cong \begin{cases}N & \text { for } q=0 \\ N / y N & \text { for } q>0 \text { odd } \\ \operatorname{ann}_{N}(y) & \text { for } q>0 \text { even }\end{cases}
$$

and

$$
\operatorname{Ext}_{S}^{q}(R, N) \cong \begin{cases}N & \text { for } q=0 \\ \operatorname{ann}_{N}(y) & \text { for } q>0 \text { odd } \\ N / y N & \text { for } q>0 \text { even. }\end{cases}
$$

Our main theorem on the vanishing of homology is the following.

\section{THEOREM 2.1}

Let $R=S /(x)$ where $S$ is a local ring and $(x, y)$ is a pair of exact zero-divisors in $S$. Furthermore, let $M$ and $N$ be $R$-modules. If there exists an integer $n \geq 2$ such that $\operatorname{Tor}_{i}^{R}(M, N)=\operatorname{Tor}_{i}^{R}(M, N / y N)=\operatorname{Tor}_{i}^{R}\left(M, \operatorname{ann}_{N}(y)\right)=0$ for $1 \leq i \leq n$, then

$$
\operatorname{Tor}_{i}^{S}(M, N) \cong \begin{cases}M \otimes_{S} N & \text { for } i=0, \\ M \otimes_{S} N / y N & \text { for } 0<i<n \text { and } i \text { odd } \\ M \otimes_{S} \operatorname{ann}_{N}(y) & \text { for } 0<i<n \text { and } i \text { even } .\end{cases}
$$

Proof

Consider the first quadrant change of rings spectral sequence (see [Rot, Theorem 10.73])

$$
\operatorname{Tor}_{p}^{R}\left(M, \operatorname{Tor}_{q}^{S}(R, N)\right) \underset{p}{\Longrightarrow} \operatorname{Tor}_{p+q}^{S}(M, N)
$$


From $(\dagger)$, the term $E_{p, q}^{2}$ is given by

$$
E_{p, q}^{2} \cong \begin{cases}\operatorname{Tor}_{p}^{R}(M, N) & \text { for } q=0 \\ \operatorname{Tor}_{p}^{R}(M, N / y N) & \text { for } q>0 \text { odd } \\ \operatorname{Tor}_{p}^{R}\left(M, \operatorname{ann}_{N}(y)\right) & \text { for } q>0 \text { even. }\end{cases}
$$

The vanishing assumptions imply that columns 1 through $n$ of the $E^{2}$-page of this spectral sequence vanish, that is, $E_{p, q}^{2}=0$ for all $q \in \mathbb{Z}$ and $1 \leq p \leq n$. Fixing such $p$ and $q$, we see that $E_{p, q}^{\infty}$ also vanishes since this term is a subquotient of $E_{p, q}^{2}$. Letting $H_{i}$ denote $\operatorname{Tor}_{i}^{S}(M, N)$ for all $i$, we have a filtration $\left\{\Phi^{j} H_{i}\right\}$ of $H_{i}$ satisfying

$$
0=\Phi^{-1} H_{i} \subseteq \Phi^{0} H_{i} \subseteq \cdots \subseteq \Phi^{i-1} H_{i} \subseteq \Phi^{i} H_{i}=H_{i},
$$

with $E_{j, i-j}^{\infty} \cong \Phi^{j} H_{i} / \Phi^{j-1} H_{i}$ for all $i$ and $j$. Thus the vanishing of $E_{p, q}^{\infty}$ implies that $\Phi^{p} H_{p+q}=\Phi^{p-1} H_{p+q}$, that is, $\Phi^{p} H_{q}=\Phi^{p-1} H_{q}$ for all $q \in \mathbb{Z}$ and $1 \leq p \leq n$.

Now consider the zeroth column of the $E^{2}$-page. For a positive $q$, the $E_{0, q^{-}}^{2}$ term is isomorphic to $M \otimes_{R} N / y N$ when $q$ is odd, and isomorphic to $M \otimes_{R}$ $\operatorname{ann}_{N}(y)$ when $q$ is even. Since $E_{p, q}^{2}=0$ for all $q \in \mathbb{Z}$ and $1 \leq p \leq n$, there is an isomorphism $E_{0, q}^{\infty} \cong E_{0, q}^{2}$ for $q \leq n-1$, giving

$$
E_{0, q}^{\infty} \cong \begin{cases}M \otimes_{S} N & \text { for } q=0, \\ M \otimes_{R} N / y N & \text { for } 0<q<n \text { and } q \text { odd, } \\ M \otimes_{R} \operatorname{ann}_{N}(y) & \text { for } 0<q<n \text { and } q \text { even. }\end{cases}
$$

But it follows from above that the equalities

$$
E_{0, q}^{\infty} \cong \Phi^{0} H_{q}=\Phi^{1} H_{q}=\cdots=\Phi^{q} H_{q}
$$

hold when $q<n$. Therefore, since $\Phi^{q} H_{q}=\operatorname{Tor}_{q}^{S}(M, N)$, we are done.

As an immediate corollary we obtain the result, for the vanishing of homology, stated in the introduction.

\section{COROLLARY 2.2}

Let $R=S /(x)$ where $S$ is a local ring and $(x, y)$ is a pair of exact zero-divisors in $S$. Furthermore, let $M$ and $N$ be $R$-modules such that $y N=0$. If there exists an integer $n \geq 2$ such that $\operatorname{Tor}_{i}^{R}(M, N)=0$ for $1 \leq i \leq n$, then $\operatorname{Tor}_{i}^{S}(M, N) \cong$ $M \otimes_{S} N$ for $0 \leq i \leq n-1$. Consequently, if $M$ and $N$ are nonzero and finitely generated, then $\operatorname{Tor}_{i}^{S}(M, N) \neq 0$ for $0 \leq i \leq n-1$.

Thus when the modules involved are finitely generated and nonzero, the corollary shows that the vanishing of homology over $R$ implies the nonvanishing of homology over $S$. This is in stark contrast to the case when $x$ is a regular element.

In certain cases we can show that the Tor's over $S$ cannot vanish irrespective of the vanishing of the Tor's over $R$. 


\section{PROPOSITION 2.3}

Let $R=S /(x)$ where $S$ is a local ring and $(x, y)$ is a pair of exact zero-divisors, both of which are minimal generators of the maximal ideal of $S$. Furthermore, let $M$ and $N$ be nonzero finitely generated $R$-modules such that $y N=0$. Then $\operatorname{Tor}_{i}^{S}(M, N) \neq 0$ for all $i \geq 0$.

\section{Proof}

Consider a minimal free resolution of $M$ over $S$ :

$$
F: \cdots \rightarrow F_{2} \stackrel{\partial_{2}}{\longrightarrow} F_{1} \stackrel{\partial_{1}}{\longrightarrow} F_{0} \rightarrow 0 .
$$

Letting $m$ denote a minimal generator of $M$, we can define the homomorphism $f: S /(x) \rightarrow M$ sending $\overline{1}$ to $m$. Because $x$ and $y$ are minimal generators of the maximal ideal of $S$, we can lift this homomorphism to a chain map

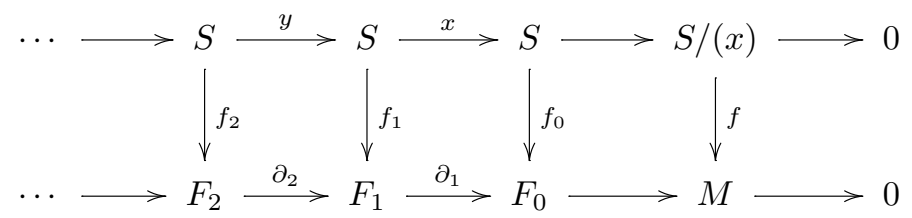

in such a way that each $f_{i}$ is a split injection. Tensoring the entire diagram with $N$ we get the commutative diagram

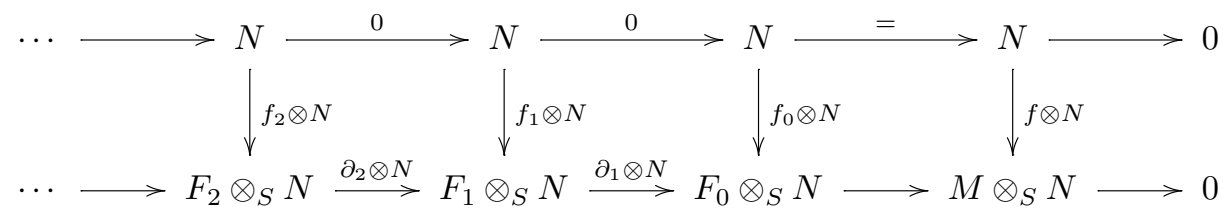

Now let $n$ be a minimal generator of $N$. Then $f_{i}(1) \otimes_{S} n$ is a minimal generator of $F_{i} \otimes_{S} N$ and, by commutativity, is in $\operatorname{ker}\left(\partial_{i} \otimes_{S} N\right)$ for all $i \geq 1$. This element is not a boundary, however, since $\partial_{i+1} \subseteq \mathfrak{m} F_{i}$, and no element in the image of $\partial_{i+1} \otimes_{S} N$ is a minimal generator of $F_{i} \otimes_{S} N$. It follows that $\operatorname{Tor}_{i}^{S}(M, N) \neq 0$ for all $i \geq 0$.

We next state the cohomological versions of Theorem 2.1 and Corollary 2.2; they are proved dually.

\section{THEOREM 2.4}

Let $R=S /(x)$ where $S$ is a local ring and $(x, y)$ is a pair of exact zero-divisors in $S$. Furthermore, let $M$ and $N$ be $R$-modules. If there exists an integer $n \geq 2$ such that $\operatorname{Ext}_{R}^{i}(M, N)=\operatorname{Ext}_{R}^{i}(M, N / y N)=\operatorname{Ext}_{R}^{i}\left(M, \operatorname{ann}_{N}(y)\right)=0$ for $1 \leq i \leq n$, then

$$
\operatorname{Ext}_{S}^{i}(M, N) \cong \begin{cases}\operatorname{Hom}_{S}(M, N) & \text { for } i=0 \\ \operatorname{Hom}_{S}\left(M, \operatorname{ann}_{N}(y)\right) & \text { for } 0<i<n \text { and } i \text { odd } \\ \operatorname{Hom}_{S}(M, N / y N) & \text { for } 0<i<n \text { and } i \text { even }\end{cases}
$$


COROLLARY 2.5

Let $R=S /(x)$ where $S$ is a local ring and $(x, y)$ is a pair of exact zero-divisors in $S$. Furthermore, let $M$ and $N$ be $R$-modules with $y N=0$. If there exists an integer $n \geq 2$ such that $\operatorname{Ext}_{R}^{i}(M, N)=0$ for $1 \leq i \leq n$, then $\operatorname{Ext}_{S}^{i}(M, N) \cong$ $\operatorname{Hom}_{S}(M, N)$ for $0 \leq i<n$. Consequently, if $N=M \neq 0$, then $\operatorname{Ext}_{S}^{i}(M, M) \neq 0$ for $0<i<n$.

\section{Complexity}

As in the previous section, we fix a local ring $S$ and a pair of exact zero-divisors $(x, y)$, and denote the local ring $S /(x)$ by $R$. In this section all modules are assumed to be finitely generated. Our aim is to compare free resolutions of modules over $R$ with those over $S$ and determine relationships involving complexities.

Given a local ring $A$ and an $A$-module $M$, there exists a (deleted) free resolution of $M$

$$
\cdots \rightarrow F_{2} \rightarrow F_{1} \rightarrow F_{0} \rightarrow 0
$$

which is minimal, that is, it appears as a direct summand of every free resolution of $M$. The cokernel of the map $F_{n+1} \rightarrow F_{n}$ is the $n$th syzygy module of $M$ and is denoted by $\Omega_{A}^{n}(M)$. Minimal free resolutions are unique up to isomorphism and hence the syzygies are uniquely determined up to isomorphism. Moreover, for every nonnegative integer $n$, the $n$th Betti number $\beta_{n}^{A}(M) \stackrel{\text { def }}{=} \operatorname{rank} F_{n}$ is a well-defined invariant of $M$. It is well known that $\operatorname{dim}_{k} \operatorname{Ext}_{A}^{n}(M, k)=\beta_{n}^{A}(M)=$ $\operatorname{dim}_{k} \operatorname{Tor}_{n}^{A}(M, k)$ for every integer $n$ where $k$ is the residue field of $A$. It is also clear that the projective dimension of $M$ is finite if and only if the Betti numbers of $M$ eventually vanish. Thus the asymptotic behavior of the Betti sequence $\beta_{0}^{A}(M), \beta_{1}^{A}(M), \beta_{2}^{A}(M), \ldots$ determines an important homological property of $M$. Following ideas from modular representation theory (see [Alp]), an invariant measuring how "fast" the Betti sequence grows was introduced by Avramov [Av2] (see also [Av1]). The complexity of $M$, denoted by $\operatorname{cx}_{A}(M)$, is defined as

$$
\operatorname{cx}_{A}(M) \stackrel{\text { def }}{=} \inf \left\{t \in \mathbb{N} \cup\{0\} \mid \exists a \in \mathbb{R} \text { such that } \beta_{n}^{A}(M) \leq a n^{t-1} \text { for all } n\right\},
$$

and measures the polynomial rate of growth of the Betti sequence of $M$. It follows from the definition that $M$ has finite projective dimension if and only if $\operatorname{cx}_{A}(M)=0$, whereas $\operatorname{cx}_{A}(M)=1$ if and only if the Betti sequence of $M$ is bounded. For an arbitrary local ring, the complexity of a module is not necessarily finite (see [Av3, Example 4.2.2]). In fact, by [Gu2, Theorem 2.3], the finiteness of the complexity for all finitely generated $A$-modules is equivalent to $A$ being a complete intersection.

We now return to our previous setting of exact zero-divisors. We first remark that every nonzero $R$-module has infinite projective dimension over $S$, that is, every such module has positive complexity over $S$. Indeed, $(\dagger)$ from the second paragraph of Section 2 shows that if $\operatorname{Tor}_{i}^{S}(R, M)=0$ for all $i \gg 0$, then $M / y M=0$. Thus $M=0$ by Nakayama's lemma. 
Over a local ring $A$, the complexity of a module equals the complexity of any of its syzygies: their minimal free resolutions are the same except at the beginning. Moreover, given a short exact sequence

$$
0 \rightarrow M_{1} \rightarrow M_{2} \rightarrow M_{3} \rightarrow 0
$$

of $A$-modules, the inequality

$$
\operatorname{cx}_{A}\left(M_{u}\right) \leq \max \left\{\operatorname{cx}_{A}\left(M_{v}\right), \mathrm{cx}_{A}\left(M_{w}\right)\right\}
$$

holds for $\{u, v, w\}=\{1,2,3\}$. This follows simply by comparing the $k$-vector space dimensions of the Tor modules in the long exact sequence

$$
\cdots \rightarrow \operatorname{Tor}_{n}^{A}\left(M_{1}, k\right) \rightarrow \operatorname{Tor}_{n}^{A}\left(M_{2}, k\right) \rightarrow \operatorname{Tor}_{n}^{A}\left(M_{3}, k\right) \rightarrow \operatorname{Tor}_{n-1}^{A}\left(M_{1}, k\right) \rightarrow \cdots,
$$

where $k$ is the residue field of $A$.

In the next proposition we use the inequality $(*)$ and prove that if $M$ is an $R$-module with $\operatorname{cx}_{S}(M) \neq 1$, then $\operatorname{cx}_{S}(M)=\operatorname{cx}_{S}\left(\Omega_{R}^{n}(M)\right)$ for all $n$. Here the assumption $\operatorname{cx}_{S}(M) \neq 1$ is necessary: the $S$-module $R$ has a minimal free resolution

$$
\cdots \rightarrow S \stackrel{y}{\rightarrow} S \stackrel{x}{\rightarrow} S \stackrel{y}{\rightarrow} S \stackrel{x}{\rightarrow} S \rightarrow 0
$$

and hence has complexity one over $S$. However its syzygies $\Omega_{R}^{n}(R)$ are all zero for $n>0$.

\section{PROPOSITION 3.1}

Let $R=S /(x)$ where $S$ is a local ring and $x$ is an exact zero-divisor in $S$. Then, for every finitely generated $R$-module $M$ with $\operatorname{cx}_{S}(M) \neq 1$, the equality $\operatorname{cx}_{S}(M)=$ $\mathrm{cx}_{S}\left(\Omega_{R}^{n}(M)\right)$ holds for all $n$.

\section{Proof}

If $\operatorname{cx}_{S}(M)=0$, then $M=0$ (see the third paragraph of Section 3). Thus the result is trivial in this case. Next suppose that $\operatorname{cx}_{S}(M)>1$. Consider the short exact sequence

$$
0 \rightarrow \Omega_{R}^{1}(M) \rightarrow F \rightarrow M \rightarrow 0,
$$

where $F$ is a free $R$-module. Since the $S$-module $R$ has complexity one so does $F$. Hence the result follows from the inequality $(*)$ and the short exact sequence considered above.

Next we will compare the Betti numbers and complexities of modules over $R$ with those over $S$. For that we first set some notations that generalize the notion of the Betti number and the complexity of a module.

Let $(A, \mathfrak{m})$ be a local ring with residue field $k$, and let $M$ and $N$ be $A$-modules with the property that $M \otimes_{A} N$ has finite length. Then, for every nonnegative integer $n$, the length of $\operatorname{Tor}_{n}^{A}(M, N)$ is finite. We define this length to be the $n$th Betti number $\beta_{n}^{A}(M, N)$ of the pair $(M, N)$, that is, $\beta_{n}^{A}(M, N) \stackrel{\text { def }}{=} \ell\left(\operatorname{Tor}_{n}^{A}(M, N)\right)$. The length complexity of the pair $(M, N)$, denoted by $\ell \operatorname{cx}_{A}(M, N)$, is then defined 
as (see [Dao2, the discussion preceding Definition 2.1])

$\ell \operatorname{cx}_{A}(M, N) \stackrel{\text { def }}{=} \inf \left\{t \in \mathbb{N} \cup\{0\} \mid \exists a \in \mathbb{R}\right.$ such that $\beta_{n}^{A}(M, N) \leq a n^{t-1}$ for all $\left.n\right\}$.

Although, letting $N=k$, we recover the Betti number and the ordinary complexity of $M$, that is,

$$
\beta_{n}^{A}(M)=\beta_{n}^{A}(M, k) \quad \text { and } \quad \operatorname{cx}_{A}(M)=\ell \operatorname{cx}_{A}(M, k),
$$

our definition for $\ell \mathrm{cx}_{A}(M, N)$ of the pair $(M, N)$ is different than the one originally given by Avramov and Buchweitz [AvBu, p. 286, para 2], where the minimal number of generators of the cohomology modules $\operatorname{Ext}_{A}^{n}(M, N)$ is used. In general there is no comparison between these two definitions of Betti numbers of the pair $(M, N)$ (see also [Dao2, Theorem 5.4]).

\section{THEOREM 3.2}

Let $R=S /(x)$ where $S$ is a local ring and $(x, y)$ is a pair of exact zero-divisors in $S$. Furthermore, let $M$ and $N$ be finitely generated $R$-modules such that $y N=0$ and $M \otimes_{R} N$ has finite length. Then, for all $n$,

$$
\beta_{n}^{R}(M, N)-\sum_{i=0}^{n-2} \beta_{i}^{R}(M, N) \leq \beta_{n}^{S}(M, N) \leq \sum_{i=0}^{n} \beta_{i}^{R}(M, N) .
$$

\section{REMARK}

We have used the convention that negative Betti numbers are zero.

Proof

As in the proof of Theorem 2.1, we consider the first quadrant change of rings spectral sequence:

$$
\operatorname{Tor}_{p}^{R}\left(M, \operatorname{Tor}_{q}^{S}(R, N)\right) \underset{p}{\Longrightarrow} \operatorname{Tor}_{p+q}^{S}(M, N) .
$$

Since $y N=0$, we see from $(\dagger)$ that the $E^{2}$-page entries are given by $E_{p, q}^{2}=$ $\operatorname{Tor}_{p}^{R}(M, N)$.

We first prove the left-hand inequality of (3.2). Fix an integer $n$, and consider the short exact sequence

$$
0 \rightarrow \Phi^{n-1} H_{n} \rightarrow \operatorname{Tor}_{n}^{S}(M, N) \rightarrow E_{n, 0}^{\infty} \rightarrow 0
$$

where $\Phi^{i} H_{n}$ is the filtration of $H_{n}$ from the proof of Theorem 2.1. Since $E_{n, 0}^{\infty}=$ $\operatorname{Ker} d_{n, 0}^{n}$, we obtain the inequality $\ell\left(\operatorname{Tor}_{n}^{S}(M, N)\right) \geq \ell\left(\operatorname{Ker} d_{n, 0}^{n}\right)$. Now for all $2 \leq$ $p \leq n$, there is an exact sequence

$$
0 \rightarrow \operatorname{Ker} d_{n, 0}^{p} \rightarrow \operatorname{Ker} d_{n, 0}^{p-1} \rightarrow \operatorname{Im} d_{n, 0}^{p} \rightarrow 0,
$$

which implies that

$$
\begin{aligned}
\ell\left(\operatorname{Ker} d_{n, 0}^{n}\right) & =\ell\left(\operatorname{Ker} d_{n, 0}^{n-1}\right)-\ell\left(\operatorname{Im} d_{n, 0}^{n}\right) \\
& =\ell\left(\operatorname{Ker} d_{n, 0}^{n-2}\right)-\left(\ell\left(\operatorname{Im} d_{n, 0}^{n-1}\right)+\ell\left(\operatorname{Im} d_{n, 0}^{n}\right)\right)
\end{aligned}
$$




$$
=\ell\left(\operatorname{Ker} d_{n, 0}^{1}\right)-\sum_{i=2}^{n} \ell\left(\operatorname{Im} d_{n, 0}^{i}\right) .
$$

For $2 \leq i \leq n$, the image of $d_{n, 0}^{i}$ is a submodule of $E_{n-i, i-1}^{i}$, and the latter is a subquotient of $E_{n-i, i-1}^{2}$. Then since $E_{n-i, i-1}^{2}=\operatorname{Tor}_{n-i}^{R}(M, N)$, there is an inequality $\ell\left(\operatorname{Im} d_{n, 0}^{i}\right) \leq \ell\left(\operatorname{Tor}_{n-i}^{R}(M, N)\right)$. Moreover, the module $E_{n, 0}^{2}$ is a subquotient of $\operatorname{Ker} d_{n, 0}^{1}$. Thus, since $E_{n, 0}^{2}=\operatorname{Tor}_{n}^{R}(M, N)$, we have that $\ell\left(\operatorname{Ker} d_{n, 0}^{1}\right) \geq$ $\ell\left(\operatorname{Tor}_{n}^{R}(M, N)\right)$. This gives

$$
\begin{aligned}
\ell\left(\operatorname{Tor}_{n}^{S}(M, N)\right) & \geq \ell\left(\operatorname{Ker} d_{n, 0}^{n}\right) \\
& =\ell\left(\operatorname{Ker} d_{n, 0}^{1}\right)-\sum_{i=2}^{n} \ell\left(\operatorname{Im} d_{n, 0}^{i}\right) \\
& \geq \ell\left(\operatorname{Tor}_{n}^{R}(M, N)\right)-\sum_{i=0}^{n-2} \ell\left(\operatorname{Tor}_{i}^{R}(M, N)\right)
\end{aligned}
$$

proving the left-hand inequality.

For the right-hand inequality, we fix an integer $n$ and consider the short exact sequence

$$
0 \rightarrow \Phi^{p-1} H_{n} \rightarrow \Phi^{p} H_{n} \rightarrow E_{p, n-p}^{\infty} \rightarrow 0
$$

for $0 \leq p \leq n$. Counting the lengths, we obtain the equalities

$$
\begin{aligned}
\ell\left(\Phi^{n} H_{n}\right) & =\ell\left(\Phi^{n-1} H_{n}\right)+\ell\left(E_{n, 0}^{\infty}\right) \\
& =\ell\left(\Phi^{n-2} H_{n}\right)+\ell\left(E_{n-1,1}^{\infty}\right)+\ell\left(E_{n, 0}^{\infty}\right) \\
& \vdots \\
& =\sum_{i=0}^{n} \ell\left(E_{i, n-i}^{\infty}\right) .
\end{aligned}
$$

Each $E_{i, n-i}^{\infty}$ is a subquotient of $E_{i, n-i}^{2}$, and so since $E_{i, n-i}^{2}=\operatorname{Tor}_{i}^{R}(M, N)$, we obtain the inequality $\ell\left(E_{i, n-i}^{\infty}\right) \leq \ell\left(\operatorname{Tor}_{i}^{R}(M, N)\right)$. Then since $\Phi^{n} H_{n}=$ $\operatorname{Tor}_{n}^{S}(M, N)$, we obtain

$$
\begin{aligned}
\ell\left(\operatorname{Tor}_{n}^{S}(M, N)\right) & =\ell\left(\Phi^{n} H_{n}\right) \\
& =\sum_{i=0}^{n} \ell\left(E_{i, n-i}^{\infty}\right) \\
& \leq \sum_{i=0}^{n} \ell\left(\operatorname{Tor}_{i}^{R}(M, N)\right)
\end{aligned}
$$

proving the right-hand inequality. 
As a consequence, using the right-hand side of the inequality (3.2), we obtain an upper bound for $\ell \mathrm{cx}_{S}(M, N)$ in terms of the complexity of $(M, N)$ over $R$.

COROLLARY 3.3

Let $R=S /(x)$ where $S$ is a local ring and $(x, y)$ is a pair of exact zero-divisors in $S$. Furthermore, let $M$ and $N$ be finitely generated $R$-modules such that $y N=0$ and $M \otimes_{R} N$ has finite length. Then $\ell \operatorname{cx}_{S}(M, N) \leq \ell \operatorname{cx}_{R}(M, N)+1$.

Proof

If $\ell \operatorname{cx}_{R}(M, N)=\infty$, then there is nothing to prove. So suppose that $\ell \operatorname{cx}_{R}(M$, $N)=c<\infty$. Then, by the definition, there exists a real number $a$ such that $\beta_{n}^{R}(M, N) \leq a n^{c-1}$ for all $n$. By Theorem 3.2, the inequality

$$
\beta_{n}^{S}(M, N) \leq \sum_{i=0}^{n} \beta_{i}^{R}(M, N) \leq \sum_{i=0}^{n} a i^{c-1} \leq(n+1) a n^{c-1}
$$

holds for all $n$. Therefore there is a real number $b$ such that $\beta_{n}^{S}(M, N) \leq b n^{c}$ for all $n$. This shows that $\ell \mathrm{cx}_{S}(M, N) \leq c+1$.

We are unaware of an example of a pair of $R$-modules for which equality holds on the left-hand side of (3.2). On the other hand, equality may occur on the righthand side. Indeed, when the exact zero-divisors $x$ and $y$ are minimal generators of the maximal ideal of $S$, Henriques and Şega [HeŞ, Theorem 1.7] proved that the equality

$$
\sum_{n=0}^{\infty} \beta_{n}^{S}(M) t^{n}=\frac{1}{1-t} \sum_{n=0}^{\infty} \beta_{n}^{R}(M) t^{n}
$$

of Poincaré series holds for every finitely generated $R$-module $M$. This gives that

$$
\beta_{n}^{S}(M)=\sum_{i=0}^{n} \beta_{i}^{R}(M) .
$$

However, when $x$ and $y$ are arbitrary, the equality of the Poincaré series stated above may fail.

\section{EXAMPLE 3.4}

Let $S=k[[x]] /\left(x^{3}\right)$ where $k$ is a field. Then $x^{2}$ is an exact zero divisor in $S$. Set $R=S /\left(x^{2}\right) \cong k[[x]] /\left(x^{2}\right)$. It can be seen that

$$
\sum_{n=0}^{\infty} \beta_{n}^{S}(k) t^{n}=\frac{1}{1-t}=\sum_{n=0}^{\infty} \beta_{n}^{R}(k) t^{n} .
$$

This example also shows that the inequality of Corollary 3.3 can be strict.

We now give an example illustrating the fact that the left-hand inequality of (3.2) does give useful lower bounds in some cases. 


\section{EXAMPLE 3.5}

Let $R=k\left[x_{1}, \ldots, x_{e}\right] /\left(x_{1}, \ldots, x_{e}\right)^{2}$, and let $M$ be a finitely generated $R$-module. Then $\Omega_{R}^{1}(M)$ is a finite-dimensional vector space over $k$ of dimension $\beta_{1}^{R}(M)$. It is easy to see that the Betti numbers of $k$ are $\beta_{n}^{R}(k)=e^{n}$. It follows that $\beta_{n}^{R}(M)=\beta_{1}^{R}(M) e^{n-1}$ for all $n \geq 1$. From the left-hand inequality of (3.2) we have

$$
\begin{aligned}
\beta_{n}^{S}(M) & \geq \beta_{n}^{R}(M)-\sum_{i=0}^{n-2} \beta_{i}^{R}(M) \\
& =\beta_{1}^{R}(M) e^{n-1}-\left(\sum_{i=1}^{n-2} \beta_{1}^{R}(M) e^{i-1}\right)-\beta_{0}^{R}(M) \\
& =\beta_{1}^{R}(M)\left(e^{n-1}-\frac{e^{n-2}-1}{e-1}\right)-\beta_{0}^{R}(M) \\
& =\beta_{1}^{R}(M)\left(\frac{e^{n}-e^{n-1}-e^{n-2}+1}{e-1}\right)-\beta_{0}^{R}(M) \\
& \geq \frac{\beta_{1}^{R}(M)}{2} e^{n-1}-\beta_{0}^{R}(M)
\end{aligned}
$$

for $e \geq 2$ and for any ring $S$ such that there exists an exact zero-divisor $x$ with $R \cong$ $S /(x)$. Note that the last inequality follows since for $e \geq 2$ we have $e^{2}-e-2 \geq 0$. Then $e^{n-2}\left(e^{2}-e-2\right) \geq 0$, which implies that $e^{n}-e^{n-1}-2 e^{n-2}+2 \geq 0$. Thus $2\left(e^{n}-e^{n-1}-e^{n-2}+1\right) \geq e^{n-1}(e-1)$, and the desired inequality follows. In particular, $R$-modules must have exponential growth over $S$ as well. As a specific example, let $S=k[x, y, z] /\left(x^{2}, y^{2}, z^{2}, y z\right)$. Then $x$ is an exact zero-divisor in $S$, and $R=S /(x) \cong k[y, z] /(y, z)^{2}$ has the form above.

When $N=k$, the assumptions that $M \otimes N$ has finite length and $y N=0$ hold automatically. Therefore, in this situation, Theorem 3.2 and Corollary 3.3 can be summarized as follows.

\section{COROLLARY 3.6}

Let $R=S /(x)$ where $S$ is a local ring and $x$ is an exact zero-divisor in $S$. Then, for every finitely generated $R$-module $M$, the inequalities

$$
\beta_{n}^{R}(M)-\sum_{i=0}^{n-2} \beta_{i}^{R}(M) \leq \beta_{n}^{S}(M) \leq \sum_{i=0}^{n} \beta_{i}^{R}(M)
$$

hold for all $n$. Consequently $\operatorname{cx}_{S}(M) \leq \operatorname{cx}_{R}(M)+1$ holds.

\section{REMARK}

It follows from [AHSS, Remark 4.4] that $R$ is a complete intersection if and only if $S$ is a complete intersection. The complexity inequality obtained in Corollary 3.6 gives a different proof for the 'only if' direction of this result: if $\operatorname{cx}_{R}(k)<\infty$, where $k$ is the residue field of $R$, then it follows from Corollary 3.6 that $\operatorname{cx}_{S}(k)<\infty$ and hence, by [Gu2, Corollary 2.5], $S$ is a complete intersection. 
Another observation related to the result stated above concerns commutative local Cohen-Macaulay Golod rings (see [Av3, Section 5.2]). Assume that $S$ is such a ring. Since a finitely generated module has infinite complexity over $S$ in the case in which it has infinite projective dimension over $S$ and $\operatorname{codepth}(S) \geq 2$ (see [Av3, Theorem 5.3.3(2)]), we conclude that $\operatorname{codepth}(S) \leq 1$. (Recall that $\mathrm{cx}_{S}(R)=1$.) Moreover, as $x$ is not regular, $\operatorname{codepth}(S)=1$. This implies that $S$ is a hypersurface and hence $R$ is a complete intersection.

As discussed in the introduction, when $x$ is regular the complexity inequality is quite different than the one obtained in Corollary 3.6. More precisely, in that case the inequalities $\operatorname{cx}_{S}(M) \leq \operatorname{cx}_{R}(M) \leq \mathrm{cx}_{S}(M)+1$ hold. In particular, the complexity of $M$ over $R$ is finite if and only if it is finite over $S$. However, in our situation, when $x$ is an exact zero-divisor, we are unable to deduce any further inequalities, such as $\mathrm{cx}_{R}(M) \leq \mathrm{cx}_{S}(M)$, from Theorem 3.2. In fact we do not know whether there exists an $R$-module $M$ with $\operatorname{cx}_{S}(M)<\infty$ and $\operatorname{cx}_{R}(M)=\infty$. We record this in the next question.

\section{QUESTION}

Let $R=S /(x)$ where $S$ is a local ring and $x$ is an exact zero-divisor in $S$. Is $\operatorname{cx}_{R}(M) \leq \operatorname{cx}_{S}(M)$ for all finitely generated $R$-modules $M$ ?

\section{Canonical elements of $\operatorname{Ext}_{R}^{2}(M, M)$ and lifting}

In this section we restrict our attention to the case where $(x, x)$ is a pair of exact zero-divisors in the local ring $S$, and $R=S /(x)$. We discuss natural chain endomorphisms of complexes over $R$, following the construction in [Eis, Section 1], and show that whether or not they are null-homotopic dictates the liftability of $R$-modules to $S$. These results generalize classical results (see, e.g., [ADS]) for lifting modules modulo a regular element to modulo the square of the regular element.

\subsection{Canonical endomorphisms of complexes}

Let

$$
F: \cdots \rightarrow F_{i+1} \stackrel{\partial_{i+1}}{\longrightarrow} F_{i} \stackrel{\partial_{i}}{\longrightarrow} F_{i-1} \rightarrow \cdots
$$

be a complex of finitely generated free $R$-modules. We let

$$
\widetilde{F}: \cdots \rightarrow \widetilde{F}_{i+1} \stackrel{\widetilde{\partial}_{i+1}}{\longrightarrow} \widetilde{F}_{i} \stackrel{\widetilde{\partial}_{i}}{\longrightarrow} \widetilde{F}_{i-1} \rightarrow \cdots
$$

denote a preimage over $S$ of the complex $F$, that is, a sequence of homomorphisms $\widetilde{\partial}_{i}: \widetilde{F}_{i} \rightarrow \widetilde{F}_{i-1}$ of free $S$-modules such that $F$ and $\widetilde{F} \otimes_{S} R$ are isomorphic $R$ complexes. From the fact that $\widetilde{\partial}_{i-1} \widetilde{\partial}_{i}\left(\widetilde{F}_{i}\right) \subseteq x \widetilde{F}_{i-2}$ for all $i$, we can write that

$$
\widetilde{\partial}_{i-1} \widetilde{\partial}_{i}=x \widetilde{s}_{i}
$$


for some homomorphism $\widetilde{s}_{i}: \widetilde{F}_{i} \rightarrow \widetilde{F}_{i-2}$. Now we define the homomorphisms $s_{i}$ : $F_{i} \rightarrow F_{i-2}$ by

$$
s_{i}=\widetilde{s}_{i} \otimes_{S} R
$$

for all $i$.

There are several properties of the $s_{i}$ 's which we should like to mention. See [Eis, Section 1] for the proofs. (Note that in our case $(x) /(x)^{2}=(x) \cong S /(x)$ is a free $S /(x)$-module.)

(a) The definition of $s_{i}$ is independent of the factorization in (3).

(b) The family $s=\left\{s_{i}\right\}$ is a chain endomorphism of $F$ of degree -2 .

(c) Let

$$
G: \cdots \rightarrow G_{i+1} \stackrel{\delta_{i+1}}{\longrightarrow} G_{i} \stackrel{\delta_{i}}{\longrightarrow} G_{i-1} \rightarrow \cdots
$$

be another complex of finitely generated free $R$-modules, and assume that there exists a chain map $f: F \rightarrow G$. Let $t=\left\{t_{i}=\widetilde{t}_{i} \otimes_{S} R: G_{i} \rightarrow G_{i-2}\right\}$ be the chain map defined by the factorizations $\widetilde{\delta}_{i-1} \widetilde{\delta}_{i}=x \widetilde{t}_{i}$ for all $i$, where $\widetilde{G}$ is a preimage over $S$ of $G$. Then the chain maps $f s$ and $t f$ are homotopic.

(d) From (c) it follows that the definition of the $s_{i}$ 's is independent, up to homotopy, of the preimage $\widetilde{F}$ of $F$ chosen in (2).

\subsection{The group $\operatorname{Ext}_{A}^{n}(M, M)$}

Let $A$ be an associative ring, and let $M$ be an $A$-module. Suppose that $F$ is a projective resolution of $M$. Then $\mathrm{H}^{n}\left(\operatorname{Hom}_{A}(F, F)\right)$ is the group of homotopy equivalence classes of chain endomorphisms of $F$ of degree $n$. For a chain endomorphism $s$ of $F$ of degree $n$, we let $[s]$ denote the class of $s$ in $\mathrm{H}^{n}\left(\operatorname{Hom}_{A}(F, F)\right)$. Let $G$ be another projective resolution of $M$ over $A$. Then the comparison maps $f: F \rightarrow G$ and $g: G \rightarrow F$ lifting the identity map on $M$ are homotopically equivalent. That is, $f g$ is homotopic to the identity map on $G$ and $g f$ is homotopic to the identity map on $F$. It follows that the map

$$
\theta_{G}^{F}: \mathrm{H}^{n}\left(\operatorname{Hom}_{A}(F, F)\right) \rightarrow \mathrm{H}^{n}\left(\operatorname{Hom}_{A}(G, G)\right)
$$

given by $[s] \mapsto[f s g]$ is an isomorphism, with inverse $\theta_{F}^{G}:[s] \mapsto[g s f]$. It is well known that this group is $\operatorname{Ext}_{A}^{n}(M, M)$ (see, e.g., [AV]).

\subsection{Canonical elements of $\operatorname{Ext}_{R}^{2}(M, M)$}

Returning to the situation where $R=S /(x)$ for the pair $(x, x)$ of exact zerodivisors, let $F$ be a free resolution of $M$ over $R$, and let $s$ be the endomorphism of $F$ defined by (4). Thus we have the element $[s] \in \mathrm{H}^{2}\left(\operatorname{Hom}_{R}(F, F)\right)$. That we call $[s]$ a canonical element of $\operatorname{Ext}_{R}^{2}(M, M)$ is reinforced by the following lemma.

\section{LEMMA 4.1}

Let $R=S /(x)$ where $S$ is a local ring and $(x, x)$ is a pair of exact zero-divisors in $S$. Suppose that $F$ and $G$ are free resolutions of a finitely generated module $M$ over $R$, that $s$ is the canonical endomorphism of $F$ as defined in (4), and that $t$ 
is the canonical endomorphism of $G$ as defined in (4). Then we have that

$$
\theta_{F}^{G}([t])=[s],
$$

where $\theta_{F}^{G}$ is the isomorphism defined in (5).

\section{Proof}

First assume that $F$ is a minimal free resolution of $M$. Then the comparison map $f: F \rightarrow G$ lifting the identity map on $M$ can be chosen to be a split injection, with splitting $g: G \rightarrow F$ also lifting the identity map on $M$. In particular, we have $g f=\mathrm{id}_{F}$, the identity map on $F$.

Denote the differential on $F$ by $\partial$, and denote that on $G$ by $\delta$. Let $(\widetilde{F}, \widetilde{\partial})$ be a preimage over $S$ of $(F, \partial)$, and let $(\widetilde{G}, \widetilde{\delta})$ be a preimage over $S$ of $(G, \delta)$. We choose preimages $\widetilde{f}$ of $f$ and $\widetilde{g}$ of $g$ over $S$ such that $\widetilde{g} \widetilde{f}=\mathrm{id}_{\widetilde{F}}$.

As $g_{i-1} \delta_{i}=\partial_{i} g_{i}$ for all $i$, there exists $u_{i}: \widetilde{G}_{i} \rightarrow \widetilde{F}_{i-1}$ such that $\widetilde{g}_{i-1} \widetilde{\delta}_{i}=\widetilde{\partial}_{i} \widetilde{g}_{i}+$ $x u_{i}$ for all $i$. Similarly, there exists $v_{i}: \widetilde{F}_{i} \rightarrow \widetilde{G}_{i-1}$ such that $\widetilde{\delta}_{i} \widetilde{f}_{i}=\widetilde{f}_{i-1} \widetilde{\partial}_{i}+x v_{i}$ for all $i$. Thus we have

$$
\begin{aligned}
x\left(\widetilde{g}_{i-2} \widetilde{t}_{i} \widetilde{f}_{i}-\widetilde{s}_{i}\right) & =\widetilde{g}_{i-2} \widetilde{\delta}_{i-1} \widetilde{\delta}_{i} \widetilde{f}_{i}-\widetilde{\partial}_{i-1} \widetilde{\partial}_{i} \\
& =\left(\widetilde{\partial}_{i-1} \widetilde{g}_{i-1}+x u_{i-1}\right)\left(\widetilde{f}_{i-1} \widetilde{\partial}_{i}+x v_{i}\right)-\widetilde{\partial}_{i-1} \widetilde{\partial}_{i} \\
& =x\left(\widetilde{\partial}_{i-1} \widetilde{g}_{i-1} v_{i}+u_{i-1} \widetilde{f}_{i-1} \widetilde{\partial}_{i}\right) .
\end{aligned}
$$

It follows that $g_{i-2} t_{i} f_{i}-s_{i}=\partial_{i-1}\left(g_{i-1} \bar{v}_{i}\right)+\left(\bar{u}_{i-1} f_{i-1}\right) \partial_{i}$ for all $i$, where $\bar{u}_{i}=$ $u_{i} \otimes_{S} R$ and $\bar{v}_{i}=v_{i} \otimes_{S} R$. We will have shown that $g t f$ is homotopic to $s$ with homotopy $h_{i}=\bar{u}_{i} f_{i}$ once we know that $\bar{u}_{i} f_{i}=g_{i-1} \bar{v}_{i}$ for all $i$. But this is easy:

$$
\begin{aligned}
x\left(u_{i} \widetilde{f}_{i}-\widetilde{g}_{i-1} v_{i}\right) & =\left(\widetilde{g}_{i-1} \widetilde{\delta}_{i}-\widetilde{\partial}_{i} \widetilde{g}_{i}\right) \widetilde{f}_{i}-\widetilde{g}_{i-1}\left(\widetilde{\delta}_{i} \widetilde{f}_{i}-\widetilde{f}_{i-1} \widetilde{\partial}_{i}\right) \\
& =-\widetilde{\partial}_{i} \widetilde{g}_{i} \widetilde{f}_{i}+\widetilde{g}_{i-1} \widetilde{f}_{i-1} \widetilde{\partial}_{i} \\
& =0
\end{aligned}
$$

hence the claim follows.

Notice that we also have $\theta_{G}^{F}([s])=[t]$ when $F$ is minimal. Therefore, for two arbitrary free resolutions $F$ and $G$ of $M, \theta_{F}^{G}([t])=[s]$ follows from composing $\theta_{F}^{G}=\theta_{L}^{G} \theta_{F}^{L}$ where $L$ is a minimal free resolution of $M$.

\subsection{Lifting}

Let $B$ be an associative ring, let $I$ be an ideal of $B$, and let $A=B / I$. Recall that a finitely generated $A$-module $M$ is said to lift to $B$ with lifting $M^{\prime}$ if there exists a finitely generated $B$-module $M^{\prime}$ such that $M \cong M^{\prime} \otimes_{B} A$ and $\operatorname{Tor}_{i}^{B}\left(M^{\prime}, A\right)=0$ for all $i \geq 1$. Similarly, a complex of finitely generated free $A$-modules

$$
F: \cdots \rightarrow F_{i+1} \stackrel{\partial_{i+1}}{\longrightarrow} F_{i} \stackrel{\partial_{i}}{\longrightarrow} F_{i-1} \rightarrow \cdots
$$

is said to lift to $B$ with lifting $\widetilde{F}$ if there exists a preimage $\widetilde{F}$ of $F$

$$
\widetilde{F}: \cdots \rightarrow \widetilde{F}_{i+1} \stackrel{\widetilde{\partial}_{i+1}}{\longrightarrow} \widetilde{F}_{i} \stackrel{\widetilde{\partial}_{i}}{\longrightarrow} \widetilde{F}_{i-1} \rightarrow \cdots
$$


such that $\widetilde{\partial}_{i-1} \widetilde{\partial}_{i}=0$ for all $i$. A close connection between these two notions of lifting will be explained in the next theorem. We also want to show that, when $R=S /(x)$ for $(x, x)$ a pair of exact zero-divisors, the triviality of the canonical element $[s]$ determines whether the module $M$ lifts to $S$.

\section{THEOREM 4.2}

Let $R=S /(x)$ where $S$ is a local ring and $(x, x)$ is a pair of exact zero-divisors in $S$. Then for every finitely generated $R$-module $M$, the following are equivalent.

(a) $M$ lifts to $S$.

(b) The canonical element $[s]$ in $\operatorname{Ext}_{R}^{2}(M, M)$ is trivial.

(c) Every free resolution of $M$ by finitely generated free $R$-modules lifts to $S$.

(d) Some free resolution of $M$ by finitely generated free $R$-modules lifts to $S$.

\section{Proof}

(a) $\Longrightarrow$ (b). Suppose that $M^{\prime}$ is a lifting of $M$ to $S$. Let

$$
\widetilde{F}: \cdots \rightarrow \widetilde{F}_{2} \stackrel{\widetilde{\partial}_{2}}{\longrightarrow} \widetilde{F}_{1} \stackrel{\widetilde{\partial}_{1}}{\longrightarrow} \widetilde{F}_{0} \rightarrow 0
$$

be a resolution of $M^{\prime}$ by finitely generated free $S$-modules. Since $\operatorname{Tor}_{i}^{S}\left(M^{\prime}, R\right)=0$ for all $i>0, F=\widetilde{F} \otimes_{S} R$ is a resolution of $M \cong M^{\prime} \otimes_{S} R$ by finitely generated free $R$-modules. Computing the endomorphism $s$ from the preimage $\widetilde{F}$ of $F$, which is exact, we see that $s$ is actually the zero endomorphism, and is therefore certainly trivial in $\operatorname{Ext}_{R}^{2}(M, M)$.

(b) $\Longrightarrow$ (c). By Lemma 4.1 the canonical element of $\operatorname{Ext}_{R}^{2}(M, M)$ is trivial regardless of which resolution by finitely generated free $R$-modules $F$ of $M$ we choose to define it. Therefore let $F$ be an arbitrary such resolution of $M$, and let $s$ be the canonical chain endomorphism defined as in (4) of the section above on canonical endomorphisms of complexes. By assumption, $s$ is homotopic to zero. Therefore there exists a homotopy $h=\left\{h_{i}\right\}$ with $h_{i}: F_{i} \rightarrow F_{i-1}$ such that $s_{i}=\partial_{i-1} h_{i}+h_{i-1} \partial_{i}$ for all $i$. Let $\widetilde{F}$ be an arbitrary preimage of $F$, with maps $\widetilde{\partial}$. Let $\widetilde{h}_{i}: \widetilde{F}_{i} \rightarrow \widetilde{F}_{i-1}$ be a preimage of $h_{i}$ for all $i$. There exists $u_{i}: \widetilde{F}_{i} \rightarrow \widetilde{F}_{i-2}$ such that $\widetilde{s}_{i}=\widetilde{\partial}_{i-1} \widetilde{h}_{i}+\widetilde{h}_{i-1} \widetilde{\partial}_{i}+x u_{i}$ for all $i$. Now consider the preimage $F^{\sharp}$ of $F$ where we take $F_{i}^{\sharp}=\widetilde{F}_{i}$ for all $i$, but we take the maps $\partial_{i}^{\sharp}=\widetilde{\partial}_{i}-x \widetilde{h}_{i}$ instead. We have

$$
\begin{aligned}
\partial_{i-1}^{\sharp} \partial_{i}^{\sharp} & =\left(\widetilde{\partial}_{i-1}-x \widetilde{h}_{i-1}\right)\left(\widetilde{\partial}_{i}-x \widetilde{h}_{i}\right) \\
& =\widetilde{\partial}_{i-1} \widetilde{\partial}_{i}-x\left(\widetilde{\partial}_{i-1} \widetilde{h}_{i}+\widetilde{h}_{i-1} \widetilde{\partial}_{i}\right) \\
& =x\left(\widetilde{s}_{i}-\widetilde{\partial}_{i-1} \widetilde{h}_{i}-\widetilde{h}_{i-1} \widetilde{\partial}_{i}\right) \\
& =0 .
\end{aligned}
$$

Thus $F^{\sharp}$ is a lifting of $F$ to $S$.

$(\mathrm{c}) \Longrightarrow(\mathrm{d})$. This is trivial. To show that $(\mathrm{d}) \Longrightarrow(\mathrm{a})$, assume that $F$ is a free resolution of $M$ by finitely generated free $R$-modules which lifts to the complex 
$\widetilde{F}$ over $S$. We claim that $\mathrm{H}_{i}(\widetilde{F})=0$ for $i \neq 0$. Indeed, if $\widetilde{\partial}_{i}(a)=0$ for some $a \in \widetilde{F}_{i}$, then $a=\widetilde{\partial}_{i+1}(b)+x c$ for some $b \in \widetilde{F}_{i+1}$ and $c \in \widetilde{F}_{i}$ by the exactness of $F$. Since $x \widetilde{\partial}_{i}(c)=0$, we have that $\widetilde{\partial}_{i}(c) \in x \widetilde{F}_{i-1}$. Again by the exactness of $F$ we have that $c=\widetilde{\partial}_{i+1}(d)+x e$ for some $d \in \widetilde{F}_{i+1}$ and $e \in \widetilde{F}_{i}$. Therefore $a=\widetilde{\partial}_{i+1}(b+x d)$. It follows that $\widetilde{F}$ is a resolution of $M^{\prime}=\mathrm{H}_{0}(\widetilde{F})$ by finitely generated free $S$-modules, and thus $M^{\prime}$ is a lifting of $M$ to $S$.

We end with an example showing that there are local rings $S$ admitting a pair of exact zero-divisors $(x, x)$, but no local ring $T$ with regular element $\widetilde{x}$ such that $S=T /\left(\widetilde{x}^{2}\right)$ and $x=\widetilde{x}+\left(\widetilde{x}^{2}\right)$. Therefore the notion of lifting modulo an exact zero-divisor is a more general notion than lifting from modulo a regular element to modulo the square of the regular element.

\section{EXAMPLE 4.3}

Let $k$ be field, let $S=k[V, X, Y, Z] / I$ where $I$ is the ideal

$$
\left(V^{2}, Z^{2}, X Y, V X+X Z, V Y+Y Z, V X+Y^{2}, V Y-X^{2}\right),
$$

and set $v=V+I$. Then $(v, v)$ is a pair of exact zero-divisors. Moreover, it is shown in [AGP] that $S$ does not have an embedded deformation. As a consequence there is no local ring $T$ and nonzero-divisor $\widetilde{V}$ of $T$ such that $S \cong T /\left(\widetilde{V}^{2}\right)$.

Acknowledgments. This paper is the result of a visit by the first and second authors to the third in October/November 2010. They thank the Department of Mathematics at the University of Texas at Arlington for their kind hospitality. The authors thank the referee for his/her careful reading, diligence, and helpful suggestions.

\section{References}

[Alp] J. L. Alperin, Periodicity in groups, Illinois J. Math. 21 (1977), 776-783, 1977. MR 0450381.

[ADS] M. Auslander, S. Ding, and Ø. Solberg, Liftings and weak liftings of modules, J. Algebra 156 (1993), 273-317. MR 1216471.

DOI 10.1006/jabr.1993.1076.

[Av1] L. L. Avramov, "Homological asymptotics of modules over local rings" in Commutative Algebra (Berkeley, CA, 1987), Math. Sci. Res. Inst. Publ. 15, Springer, New York, 1989, 33-62. MR 1015512.

DOI 10.1007/978-1-4612-3660-3_3.

[Av2] _ Modules of finite virtual projective dimension, Invent. Math. 96 (1989), 71-101. MR 0981738. DOI 10.1007/BF01393971.

[Av3] _ , "Infinite free resolutions" in Six Lectures on Commutative Algebra (Bellaterra 1996), Progr. Math. 166, Birkhäuser, Basel, 1998, 1-118.

MR 1648664. 
$[\mathrm{AvBu}]$ L. L. Avramov and R.-O. Buchweitz, Support varieties and cohomology over complete intersections, Invent. Math. 142 (2000), 285-318. MR 1794064. DOI 10.1007/s002220000090.

[AGP] L. L. Avramov, V. N. Gasharov, and I. V. Peeva, A periodic module of infinite virtual projective dimension, J. Pure Appl. Algebra 62 (1989), 1-5. MR 1026870. DOI 10.1016/0022-4049(89)90016-9.

[AHŞ] L. L. Avramov, I. B. Henriques, and L. M. Şega, Quasi-complete intersection homomorphisms, Pure Appl. Math. Q. 9 (2013), 1-9.

[AV] L. L. Avramov and O. Veliche, Stable cohomology over local rings, Adv. Math. 213 (2007), 93-139. MR 2331239. DOI 10.1016/j.aim.2006.11.012.

[Be] P. A. Bergh, On the vanishing of (co)homology over local rings, J. Pure Appl. Algebra 212 (2008), 262-270. MR 2355028.

DOI 10.1016/j.jpaa.2007.05.001.

[Dao1] H. Dao, Some observations on local and projective hypersurfaces, Math. Res. Lett. 15 (2008), 207-219. MR 2385635. DOI 10.4310/MRL.2008.v15.n2.a1.

[Dao2] - Asymptotic behaviour of Tor over complete intersections and applications, preprint, arXiv:0710.5818v1 [math.AC]

[Eis] D. Eisenbud, Homological algebra on a complete intersection, with an application to group representations, Trans. Amer. Math. Soc. 260 (1980), no. 1, 35-64. MR 0570778. DOI 10.2307/1999875.

[Gu1] T. H. Gulliksen, A change of ring theorem with applications to Poincaré series and intersection multiplicity, Math. Scand. 34 (1974), 167-183. MR 0364232.

[Gu2] - On the deviations of a local ring, Math. Scand. 47 (1980), 5-20. MR 0600076.

[HeŞ] I. B. D. A. Henriques and L. M. Şega, Free resolutions over short Gorenstein local rings, Math. Z. 267 (2011), 645-663. MR 2776052. DOI 10.1007/s00209-009-0639-z.

[Jo1] D. A. Jorgensen, Tor and torsion on a complete intersection, J. Algebra 195 (1997), 526-537. MR 1469637. DOI 10.1006/jabr.1997.7064.

[Jo2] C Complexity and Tor on a complete intersection, J. Algebra 211 (1999), 578-598. MR 1666660. DOI 10.1006/jabr.1998.7743.

[Rot] J. J. Rotman, An Introduction to Homological Algebra, 2nd ed., Universitext, Springer, New York, 2009. MR 2455920. DOI 10.1007/b98977.

Bergh: Institutt for matematiske fag, Norges teknisk-naturvitenskapelige universitet, N-7491 Trondheim, Norway; bergh@math.ntnu.no

Celikbas: Department of Mathematics, University of Kansas, Lawrence, Kansas 66045, USA; current: Department of Mathematics, University of Missouri, Columbia, Missouri 65211, USA; celikbaso@missouri.edu

Jorgensen: Department of Mathematics, University of Texas at Arlington, Arlington, Texas 76019, USA; djorgens@uta.edu 